QCD Evolution Workshop 2013

International Journal of Modern Physics: Conference Series

Vol. 25 (2014) 1460012 (8 pages)

(C) The Author

DOI: $10.1142 / \mathrm{S} 201019451460012 \mathrm{X}$

\title{
ESTIMATING THE SIGN AND SIZE OF THE TRANSVERSE SINGLE TARGET-SPIN ASYMMETRY IN INCLUSIVE DIS*
}

\author{
MARC SCHLEGEL \\ Institute for Theoretical Physics \\ University of Tübingen \\ Auf der Morgenstelle 14 \\ D-72076 Tübingen, Germany \\ marc.schlegel@uni-tuebingen.de
}

Published 2 January 2014

\begin{abstract}
The single-spin asymmetry of unpolarized leptons scattering deep-inelastically off transversely polarized nucleons is discussed. This observable is generated by a two-photon exchange between lepton and nucleon. In a partonic description of the asymmetry the non-perturbative part is given in terms of multiparton correlations: quark-gluon correlation functions and quark-photon correlation functions. Using input from models and data fits for these twist-3 correlation functions, estimates for the single-spin asymmetries for a proton and a neutron are presented.
\end{abstract}

Keywords: Single-Spin Asymmetry; Two-Photon Exchange; Twist-3.

PACS numbers: 13.60.Hb, 13.88.+e

\section{Introduction}

One of the most fundamental and basic processes in hadronic physics is the deepinelastic scattering (DIS) of leptons off nucleons, $\mathrm{l}(l)+\mathrm{N}(P) \rightarrow \mathrm{l}\left(l^{\prime}\right)+\mathrm{X}$. Historically, the experimental and theoretical analyses of this simple reaction has provided valuable information on the partonic structure of the nucleon and has led to important progress in the understanding of the strong interactions - and ultimately to the developement of Quantum Chromodynamics (QCD). Single-spin observables in inclusive DIS with either the lepton or nucleon being transversely polarized strictly vanish due to time-reversal invariance for a single photon exchange [1]. This argument fails if two (or more) photons are exchanged between lepton and nucleon.

Experimentally, the single-spin asymmetry (SSA) for a transversely polarized nucleon, denoted by $A_{U T}$, was already measured in 1970 in the resonance region [2].

*This is an Open Access article published by World Scientific Publishing Company. It is distributed under the terms of the Creative Commons Attribution 3.0 (CC-BY) License. Further distribution of this work is permitted, provided the original work is properly cited. 
A result consistent with zero within an error of about $10^{-2}$ was obtained. A recent measurement of the single-spin asymmetry (SSA) for transversely polarized protons was performed by the HERMES collaboration [3], and again a result consistent with zero was found within an error of about $10^{-3}$. Interestingly, preliminary data taken from (ongoing) precision measurements of $A_{U T}$ on a polarized ${ }^{3} \mathrm{He}$ target at Jefferson Lab - mimicking a polarized neutron target - seem to indicate a non-zero effect [4].

A theoretical description of the SSA $A_{U T}$ in a partonic picture needs to deal with two distinctive and complementary physical situations: The exchange of two photons between the lepton and either (i) one single quark or (ii) two different quarks. The asymmetry has been studied in Refs. $[5,6,7,8]$ for massless quarks. It was found that this observable generically behaves like $M / Q$ where $M$ denotes the nucleon mass, $Q^{2}=-q^{2}$, and $q=l-l^{\prime}$ the 4-momentum transfer to the nucleon. Thus the asymmetry is a power suppressed ('twist-3') observable, and can be expressed in terms of multipartonic non-perturbative quark-gluon (scenario (i)) and quark-photon (scenario (ii) correlation functions. Effects of a finite quark mass proportional to the transversity distribution $h_{1}^{q}(x)$ are also relevant for scenario (i) and have been studied in Ref. [9].

\section{2. $A_{U T}$ in a Partonic Picture}

The DIS differential cross section can be analyzed in terms of the commonly used DIS variables that are defined as $x_{B}=Q^{2} /(2 P \cdot q)$ and $y=P \cdot q / P \cdot l$. For the description of $A_{U T}$ a transverse (to the lepton plane) spin vector $S_{T}$ of a polarized nucleon is needed. An azimuthal angle $\phi_{s}$ between $S_{T}$ and the lepton plane determines the spatial orientation of $S_{T}$.

An analysis of the SSA $A_{U T}$ in inclusive DIS in a partonic picture has to be performed at subleading twist accuracy $[5,7,8]$. This requires the introduction of typical hadronic matrix elements of certain partonic operators that encode non-trivial correlations of the transverse nucleon spin and the transverse partonic motion [10], as well as multipartonic correlations $[11,8]$. However, the effects of transverse partonic motion and multipartonic correlations are not independent. In fact, they can related to each other by means of the QCD-equation of motion (EOM) [10]. An additional dependence originates from the relation between the Sivers function and the so-called Qiu-Sterman matrix element [13]. If one applies the twist-3 factorization formalism of Ref. [12] to the SSA $A_{U T}$ all of these hadronic matrix elements are to be convoluted with corresponding partonic hard cross sections, and eventually summed up (cf. [7]).

The hard cross sections relevant for scenario (i) are calculated in perturbation theory to $\mathcal{O}\left(\alpha^{3}\right)$ to obtain a non-zero result. This includes interferences of real lepton-quark(\& gluon) scattering amplitudes describing the radiation of a photon emitted by either the lepton or the quark. Such real contributions typically contain phase space integrations. Interferences from virtual two-photon-exchange one-loop 
diagrams and single photon exchange diagrams may also contribute. The various hard cross sections can be combined by application of QCD-EOM inspired relations between effects of transverse partonic motion and multipartonic correlations, and eventually the soft divergences indicated by poles in $1 / \varepsilon$ cancel [7].

The hard cross sections can be computed along the same lines for scenario (2). To leading order only tree-level diagrams interfere without phase space integrations [8]. Hence, no soft divergences appear in intermediate steps of the calculation.

Adding the results of Refs. [7, 9, 8] leads to the following parton picture formula for the single transverse spin dependent DIS cross section at $\mathcal{O}\left(\alpha_{\mathrm{em}}^{3}\right)$,

$$
\begin{aligned}
E^{\prime} \frac{\mathrm{d} \sigma_{U T}}{\mathrm{~d}^{3} l^{\prime}}= & -\left|S_{T}\right| \sin \phi_{s} \frac{4 \alpha_{\mathrm{em}}^{3}}{y Q^{4}} \frac{M}{Q} \frac{x_{B} y}{\sqrt{1-y}} \\
& \times \sum_{q}\left[e_{q}^{3} \int_{0}^{1} d x\left(\hat{C}_{+}\left(x, x_{B}, y\right) G_{F}^{q}\left(x_{B}, x\right)+\hat{C}_{-}\left(x, x_{B}, y\right) \tilde{G}_{F}^{q}\left(x_{B}, x\right)\right)\right. \\
& +e_{q}^{3}(1-y) \frac{m_{q}}{M} h_{1}^{q}\left(x_{B}\right)+\frac{2-y}{2 y}\left(1-y+\frac{y^{2}}{2}\right) \\
& \left.\times e_{q}^{2}\left(1-x_{B} \frac{\mathrm{d}}{\mathrm{d} x_{B}}\right) G_{F}^{\gamma, q}\left(x_{B}, x_{B}\right)+\mathcal{O}\left(\frac{M^{2}}{Q^{2}}\right)\right]
\end{aligned}
$$

The perturbative coefficient functions $\hat{C}_{ \pm}$in Eq. (1) are integrable distributions and their functional form is given in Ref. [7]. Assuming that the non-perturbative quarkgluon correlation functions $G_{F}^{q}\left(x, x^{\prime}\right)$ and $\tilde{G}_{F}^{q}\left(x, x^{\prime}\right)^{\text {a }}$ are analytic the $x$-integral in (1) is well-defined. In addition the finite quark mass term of Ref. [9, 8] has been added to Eq. (1) as well as the contribution of Ref. [8] describing scenario (ii) where the two photons couple to different quarks. The latter term involves a quark-photon correlation function $G_{F}^{\gamma}$ b.

One can modify the form of the perturbative coefficients $\hat{\mathcal{C}}_{ \pm}$in Eq. (1) in order to isolate specifically contributions proportional to the so-called "Soft-Gluon Pole" matrix element $G_{F}^{q}(x, x)$ from contributions determined by "non-diagonal" quarkgluon correlations functions $G_{F}^{q}\left(x, x^{\prime}\right)$, with $x \neq x^{\prime}$. Doing so yields,

$$
\begin{aligned}
& E^{\prime} \frac{\mathrm{d} \sigma_{U T}}{\mathrm{~d}^{3} l^{\prime}}=-\left|S_{T}\right| \sin \phi_{s} \frac{4 \alpha_{\mathrm{em}}^{3}}{y Q^{4}} \frac{M}{Q} \frac{x_{B} y}{\sqrt{1-y}} \times \\
& \quad\left[\hat{F}\left(x_{B}, y\right) G_{F}\left(x_{B}, x_{B}\right)+\int_{0}^{1} \mathrm{~d} x \frac{\hat{F}_{+}\left(\frac{x}{x_{B}}, y\right) G_{F}\left(x_{b}, x\right)+\hat{F}_{-}\left(\frac{x}{x_{B}}, y\right) \tilde{G}_{F}\left(x_{b}, x\right)}{\left(x-x_{B}\right)_{+}}\right. \\
& \left.\quad+(1-y) h_{1}\left(x_{B}\right)+\frac{2-y}{2 y}\left(1-y+\frac{y^{2}}{2}\right)\left(1-x_{B} \frac{\mathrm{d}}{\mathrm{d} x_{B}}\right) G_{F}^{\gamma}\left(x_{B}, x_{B}\right)+\mathcal{O}\left(\frac{M^{2}}{Q^{2}}\right)\right] .
\end{aligned}
$$

\footnotetext{
${ }^{a}$ Definitions in terms of hadronic matrix elements for both functions can be found in Ref. [11]. ${ }^{\mathrm{b}}$ Notice a redefinition $G_{F}^{\gamma}(x, x) \equiv \frac{4 \pi}{\alpha_{\mathrm{em}}} F_{F T}(x, x)$ of the object $F_{F T}$ introduced in [8]. The redefinition suggested in [7] is incorrect. Also note that the factor $1-y+y^{2} / 2$ in the last term in Eq. (1) is missing in [7].
} 
Here, the fractional quark charges $e_{q}$ are hidden in the following notations,

$$
\begin{aligned}
G_{F}\left(x, x^{\prime}\right) & \equiv \sum_{q} e_{q}^{3} G_{F}^{q}\left(x, x^{\prime}\right) ; \tilde{G}_{F}\left(x, x^{\prime}\right) \equiv \sum_{q} e_{q}^{3} \tilde{G}_{F}^{q}\left(x, x^{\prime}\right) ; \\
h_{1}(x) & \equiv \sum_{q} e_{q}^{3} \frac{m_{q}}{M} h_{1}^{q}(x) ; G_{F}^{\gamma}\left(x, x^{\prime}\right) \equiv \sum_{q} e_{q}^{2} G_{F}^{\gamma, q}\left(x, x^{\prime}\right) .
\end{aligned}
$$

In addition the usual + - prescription is used,

$$
\int_{x}^{1} \mathrm{~d} z \frac{f(z)}{(1-z)_{+}}=\int_{x}^{1} \mathrm{~d} z \frac{f(z)-f(1)}{1-z} .
$$

The perturbative functions $\hat{F}, \hat{F}_{+}$and $\hat{F}_{-}$read

$$
\begin{aligned}
\hat{F}\left(x_{B}, y\right)= & \left(\ln y^{2}+2(1-y)(2-y)+1-y+\frac{y^{2}}{2}\right) \ln \frac{1-x_{B}}{x_{B}}-\left(1-\frac{y}{2}\right)^{2}, \\
\hat{F}_{+}(\alpha, y)= & \left(1-y+\frac{y^{2}}{2}\right) \theta(\alpha-1)+\frac{\alpha+1}{\alpha} \ln y+(1-y)^{2} \frac{\ln \alpha}{\alpha-1}+\frac{\ln (y+\alpha(1-y))}{\alpha(\alpha-1)} \\
& +\frac{1}{2}(1-y) \frac{1+2 y-y^{2}+\alpha\left(3-4 y+y^{2}\right)}{y+\alpha(1-y)}, \\
\hat{F}_{-}(\alpha, y)= & \left(1-y+\frac{y^{2}}{2}\right) \theta(\alpha-1)+\frac{\alpha-1}{\alpha} \ln y+(1-y)^{2} \frac{\ln \alpha}{\alpha-1}-\frac{\ln (y+\alpha(1-y))}{\alpha(\alpha-1)} \\
& +\frac{1}{2}(1-y) \frac{-1+2 y+y^{2}+\alpha\left(1-y^{2}\right)}{y+\alpha(1-y)} .
\end{aligned}
$$

The SSA $A_{U T}$ can be computed from (2) in the target rest frame as suggested in Ref. [8]. In this frame the polarized target is at rest while the lepton beam points towards the negative $z$-direction. The lepton plane then determines the $x z$-plane, with the outgoing lepton momentum in the positive $x$-direction. The $y$-direction is fixed by the cross product $\hat{e}_{y}=\hat{e}_{z} \times \hat{e}_{x}$. The SSA is proportional to the component of the target spin vector in $y$-direction, $S_{N}^{y}$, in this frame, and is defined in the following way $\left(\mathrm{d} \sigma=E^{\prime} \mathrm{d} \sigma / \mathrm{d}^{3} k^{\prime}\right)$,

$$
A_{U T}=\frac{\left.\mathrm{d} \sigma_{U T}\left(S_{N}^{y}=+1\right)-d \sigma_{U T}\left(S_{N}^{y}=-1\right)\right)}{2 \mathrm{~d} \sigma_{U U}}
$$

with the well-known parton model result for the unpolarized cross section [10],

$$
\mathrm{d} \sigma_{U U}=\frac{4 \alpha_{\mathrm{em}}^{2}}{Q^{4} y}\left(1-y+\frac{y^{2}}{2}\right) \sum_{q} e_{q}^{2} x_{B} f_{1}^{q}\left(x_{B}\right),
$$

with $f_{1}^{q}$ the unpolarized collinear parton distribution and $f_{1}(x) \equiv \sum_{q} e_{q}^{2} f_{1}^{q}(x)$. 
The leading order (in $\mathcal{O}\left(\alpha_{s}\right)$ ) expression at twist-3 for the asymmetry is then explicitly given in the target rest frame as follows,

$$
\begin{gathered}
A_{U T}\left(x_{B}, y, Q^{2}\right)=-\alpha_{\text {em }} \frac{M}{Q} \frac{y}{\sqrt{1-y}\left(1-y+\frac{y^{2}}{2}\right)}\left[\hat{F}\left(x_{B}\right) \frac{G_{F}\left(x_{B}, x_{B}\right)}{f_{1}\left(x_{B}\right)}\right. \\
+\frac{1}{f_{1}\left(x_{B}\right)} \int_{0}^{1} \mathrm{~d} x \frac{\hat{F}_{+}\left(\frac{x}{x_{B}}, y\right) G_{F}\left(x_{B}, x\right)+\hat{F}_{-}\left(\frac{x}{x_{B}}, y\right) \tilde{G}_{F}\left(x_{B}, x\right)}{\left(x-x_{B}\right)_{+}} \\
\left.+\frac{2-y}{2 y}\left(1-y+\frac{y^{2}}{2}\right) \frac{\left(1-x_{B} \frac{\mathrm{d}}{\mathrm{d} x_{B}}\right) G_{F}^{\gamma}\left(x_{B}, x_{B}\right)}{f_{1}\left(x_{B}\right)}+(1-y) \frac{h_{1}\left(x_{B}\right)}{f_{1}\left(x_{B}\right)}+\mathcal{O}\left(\frac{M^{2}}{Q^{2}}\right)\right] \\
\equiv A_{U T}^{\text {Siv }}+A_{U T}^{\text {non }-\operatorname{diag}}+A_{U T}^{\gamma}+A_{U T}^{m_{q}} .
\end{gathered}
$$

Eq. (8) suggests four different mechanisms that contribute to the single spin asymmetry in inclusive DIS. The first term, $A_{U T}^{\text {Siv }}$, may be interpreted as a Sivers-effect [14] in inclusive DIS because it is generated by a non-vanishing soft-gluon pole martix element $G_{F}(x, x)=\frac{2}{\pi} f_{1 T}^{\perp(1)}(x)$ which in turn can be expressed in terms of the first $k_{T}$-moment of the Sivers function $f_{1 T}^{\perp}[13]^{\mathrm{c}}$. The second term, $A_{U T}^{\text {non-diag }}$, mainly receives contributions from quark-gluon correlation functions $G_{F}\left(x, x^{\prime}\right)$ and $\tilde{G}_{F}\left(x, x^{\prime}\right)$ that are to be evaluated in those regions of their support that are away from the diagonal $x=x^{\prime}$. In particular the dependence on the soft-gluon pole matrix element $G_{F}(x, x)$ is minimized due to the + - description of the denominator in the integrand. Applying the same reasoning as for $A_{U T}^{\mathrm{Siv}}$, the third term, $A_{U T}^{\gamma}$, may be interpreted as a photonic Sivers effect because of the dependence on a soft-photon pole matrix element $G_{F}^{\gamma}(x, x)$. The fourth term, $A_{U T}^{m_{q}}$, displays a chiral-odd helicity flip of the scattered quark induced by a non-vanishing quark mass [9].

\section{Numerical Predictions for the Transverse Target SSA by Means of Models and Fits from Data}

In order to utilize Eq. (8) to estimate the sign and size of the four contributions to the transverse target spin asymmetry $A_{U T}$ on a proton and neutron one needs information on the full support of the non-perturbative quark-gluon correlation functions $G_{F}^{q}\left(x, x^{\prime}\right), \tilde{G}_{F}^{q}\left(x, x^{\prime}\right)$, as well as the quark-photon correlation function $G_{F}^{\gamma, q}(x, x)$ in the soft photon limit $x^{\prime}=x$ and the transversity distribution $h_{1}^{q}(x)$. Currently, only extractions from data exist for the Soft Gluon Pole matrix element $G_{F}^{q}(x, x)[16,17,18]$ and the transversity distribution [19]. In order to estimate the contributions $A_{U T}^{\text {non-diag }}$ and $A_{U T}^{\gamma}$ one has to rely on models. A recent model calculation gives predictions for $G_{F}^{q}$ and $\tilde{G}_{F}^{q}$ on the full support $x \neq x^{\prime}$ [15]. In this work the twist-3 quark-gluon correlation functions are expressed in terms on non-valence-like light cone wave functions, and analytical results at a scale $\mu_{0}=$ $1 \mathrm{GeV}$ were obtained. One specific feature of this model is that $G_{F, \text { Model }}^{q}\left(x, x, \mu_{0}\right)=$

${ }^{\mathrm{c}}$ Note that $G_{F}^{q}\left(x, x^{\prime}\right)=G_{F}^{q}\left(x^{\prime}, x\right)$ and $\tilde{G}_{F}^{q}\left(x, x^{\prime}\right)=-\tilde{G}_{F}^{q}\left(x^{\prime}, x\right)$. Hence, $\tilde{G}_{F}^{q}(x, x)=0$. 
0 due to the absence of final state interactions. This is in obvious contradiction to parametrizations from data for $G_{F}^{q}(x, x)[16,17,18]$, and the model does not properly describe the physics of $G_{F}^{q}\left(x, x^{\prime}\right)$ in a small interval around $x^{\prime} \sim x$. As mentioned before the SSA in Eq. (8) is written in a form where the dependence of the term $A_{U T}^{\text {non-diag }}$ on the region of the support of the function $G_{F}$ near the diagonal $x=x^{\prime}$ is minimized because of the + - prescription. Hence the model of Ref. [15] may be used as input for $A_{U T}^{\text {non-diag }}$ to probe the physics in the region $x \neq x^{\prime}$.

In Ref. [8] both the soft-photon pole matrix element $G_{F}^{\gamma}(x, x)$ and the soft-gluon pole matrix element $G_{F}(x, x)$ were calculated in a simple diquark spectator model. Within this model one obtains the following relations between these twist-3 objects,

$$
\begin{aligned}
& G_{F}^{\gamma, u / p}(x, x)=-\frac{2 \pi}{3 C_{F} \alpha_{s}} G_{F}^{u / p}(x, x) ; G_{F}^{\gamma, d / p}(x, x)=-\frac{8 \pi}{3 C_{F} \alpha_{s}} G_{F}^{d / p}(x, x) ; \\
& G_{F}^{\gamma, u / n}(x, x)=+\frac{4 \pi}{3 C_{F} \alpha_{s}} G_{F}^{d / p}(x, x) ; G_{F}^{\gamma, d / p}(x, x)=-\frac{2 \pi}{3 C_{F} \alpha_{s}} G_{F}^{u / p}(x, x) .
\end{aligned}
$$

Here, $C_{F}=4 / 3$ and $\alpha_{s}$ the strong coupling taken at some non-perturbative scale. While the running of the strong coupling is not well-known in the non-perturbative region, some value $\alpha_{s}=1$ is chosen which roughly corresponds to the running coupling at two-loop accuracy at a scale $\mu=1 \mathrm{GeV}$ and three active flavors, i.e., $\alpha_{s, 2-\text { loop }}^{n_{f}=3}(1 \mathrm{GeV}) \sim 1$. It is then possible to give predictions for $A_{U T}^{\gamma}$ using the model relations $(9)$ in connection with the fits for the soft-gluon pole matrix element $G_{F}(x, x)[8]$.

The quark mass effects $A_{U T}^{m_{q}}$ can be estimated using the fits of Ref. [19] for the transversity distribution. If current quark masses are assumed the effects $A_{U T}^{m_{q}}$ become negligible. In Ref. [9] it was argued that the quark helicity can be flipped by interactions with the non-perturbative vacuum, promoting the values of the quark masses $m_{q}$ to constituent quark masses of about $0.3 \mathrm{GeV}$. While this reasoning is well beyond the perturbative QCD picture of Eq. (8), one may understand $A_{U T}^{m_{q}}$ with constituent quark masses as an upper bound for chiral-odd quark mass effects.

The model predictions for $A_{U T}$ are shown in Fig. 1. In this plot fixed target kinematics have been used for an electron beam energy $E=12 \mathrm{GeV}$ (JLab12 kinematics). The hard scale is fixed at $Q=1 \mathrm{GeV}$. A missing mass $W=(P+q)^{2}>$ $4 \mathrm{GeV}^{2}=W_{\min }$ was assumed to ensure that the asymmetry is probed in the DIS region. This defines a maximal Bjorken-variable $x_{B, \max }=Q^{2} /\left(Q^{2}+W_{\min }-M^{2}\right) \sim$ 0.25 for $Q=1 \mathrm{GeV}$. For a fixed scale the energy transfer from the electron to the nucleon $y$ varies with $x_{B}$, that is, $y=Q^{2} /\left(2 M E x_{B}\right)$. Typical experimental values $y \sim 0.4-0.6$ are probed at $x_{B} \sim 0.1$ at $Q=1 \mathrm{GeV}$. In the lower $x_{B}$-region of $x_{B} \sim 0.05$ the variable $y$ which describes how much energy is transferred from the lepton to the nucleon approaches 1.

According to Fig. 1 the contribution $A_{U T}^{\gamma}$ is dominant for a proton and neutron at larger $x_{B}$. In particular for a neutron unexpectedly large effects at the percent 

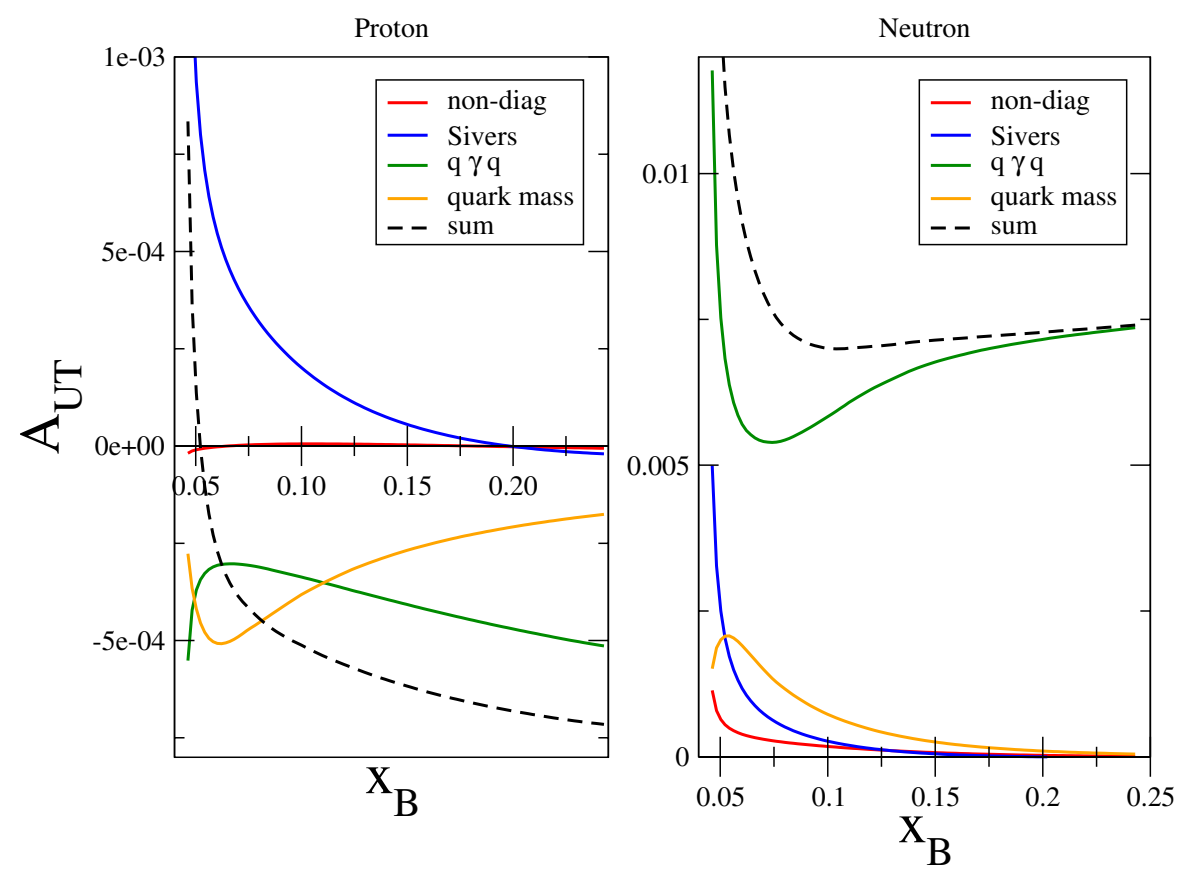

Fig. 1. Estimates for the various contributions of Eq. (8) to the asymmetry $A_{U T}$ on a proton (left) and a neutron (right) in JLab12 kinematics at a fixed low scale $Q=1 \mathrm{GeV}$.

level are predicted. This feature has already been observed in Ref. [8] .

At lower $x_{B}$ the "Sivers effect" $A_{U T}^{\text {Siv }}$ is roughly of the same size for a proton compared to $A_{U T}^{\gamma}$, however with opposite sign. In particular, the parameterization [17] was used to predict the effects $A_{U T}^{\mathrm{Siv}}$ for a proton and neutron in Fig. 1.

The model [15] suggests that the contributions $A_{U T}^{\text {non-diag }}$ are small for a proton and neutron. This was observed already in Ref. [20].

\section{Summary}

The single-spin asymmetry of leptons scattering deep-inelastically off transversely polarized nuleons was discussed in a partonic picture. It was argued that four different types of mechanisms contribute to this observable. By means of models and data fits as input for non-perturbative twist-3 multiparton correlation function numerical predictions for the four types of contributions are presented specifically for JLab12 at fixed scale $Q=1 \mathrm{GeV}$. One may get further information about this observable by varying the scale $Q$. This would allow to present predictions also for a future electron-ion-collider (EIC). However, in order to get a realistic picture of the scale

\footnotetext{
${ }^{\mathrm{d}}$ Note that in this work a different choice for the running coupling, $\alpha_{s}$, at one-loop accuracy was used leading to larger asymmetries.
} 
dependence, the evolution of the twist-3 correlation function has to be taken into account. Even though a numerical implementation of twist-3 evolution has been put forward recently [21] it goes beyond the scope of these proceedings contributions and is left for future work.

\section{References}

1. N. Christ, T.D. Lee, Phys. Rev. 143, 1310-1321 (1966).

2. J.R. Chen et al., Phys. Rev. Lett. 21, 1279 (1968); J.A. Appel et al., Phys. Rev. D1, 1285 (1970); S. Rock et al., Phys. Rev. Lett. 24, 748 (1970).

3. A. Airapetian et al., Phys. Lett. B 682, 351 (2010).

4. Jefferson Lab experiment E07-013, Spokespersons: T.D. Averett, T. Holmstrom, X. Jiang; J.M. Katich, Ph.D. thesis, The College of William and Mary, 2011.

5. A. Metz, M. Schlegel, K. Goeke, Phys. Lett. B 643, 319 (2006).

6. M. Schlegel, A. Metz, AIP Conf.Proc. 1149, 543 (2009).

7. M. Schlegel, Phys. Rev. D 87, 034006 (2013).

8. A. Metz, D. Pitonyak, A. Schafer, M. Schlegel, W. Vogelsang, J. Zhou, Phys. Rev. D 86, 094039 (2012).

9. A. Afanasev, M. Strikman, C. Weiss, Phys. Rev. D 77, 014028 (2008).

10. A. Bacchetta, M. Diehl, K. Goeke, A. Metz, P.J. Mulders, M. Schlegel, JHEP 0702, 093 (2007).

11. A. Accardi, A. Bacchetta, W. Melnitchouk, M. Schlegel, JHEP 0911, 093 (2009).

12. R.K. Ellis, W. Furmanski, R. Petronzio, Nucl. Phys. B 207, 1 (1982); Nucl. Phys. B 212, 29 (1983).

13. D. Boer, P.J. Mulders, F. Pijlman, Nucl. Phys. B 667, 201 (2003); J.W. Qiu, G.F. Sterman, Phys. Rev. Lett. 67, 2264 (1991); Nucl. Phys. B 378, 52 (1992).

14. D. Sivers, Phys. Rev. D 41, 83 (1990); Phys. Rev. D 43, 261 (1991).

15. V.M. Braun, T. Lautenschlager, A.N. Manashov, B. Pirnay, Phys. Rev. D 83, 094023 (2011).

16. C. Kouvaris et al., Phys. Rev D74, 114013 (2006).

17. A. Anselmino et al., Eur. Phys. J. A 39, 89 (2009).

18. Z.-B. Kang, A. Prokudin, Phys. Rev. D 85, 074008 (2012).

19. M. Anselmino et al., Phys. Rev. D 87, 094019 (2013); Phys. Rev. D 75, 054032 (2007).

20. M. Schlegel, arXiv:1303.0978 [hep-ph] (2013).

21. B.M. Pirnay, arXiv:1307.1272 [hep-ph] (2013). 